\title{
ESO-Based Fuzzy Sliding-Mode Control for a 3-DOF Serial-Parallel Hybrid Humanoid Arm
}

\author{
Yueling Wang, Runjie Shi, and Hongbin Wang \\ Institute of Electrical Engineering, Yanshan University, Qinhuangdao 066004, China \\ Correspondence should be addressed to Yueling Wang; yuelingw@ysu.edu.cn
}

Received 15 March 2014; Accepted 27 May 2014; Published 3 August 2014

Academic Editor: Onur Toker

Copyright (C) 2014 Yueling Wang et al. This is an open access article distributed under the Creative Commons Attribution License, which permits unrestricted use, distribution, and reproduction in any medium, provided the original work is properly cited.

\begin{abstract}
This paper presents a unique ESO-based fuzzy sliding-mode controller (FSMC-ESO) for a 3-DOF serial-parallel hybrid humanoid arm (HHA) for the trajectory tracking control problem. The dynamic model of the HHA is obtained by Lagrange method and is nonlinear in dynamics with inertia uncertainty and external disturbance. The FSMC-ESO is based on the combination of the sliding-mode control (SMC), extended state observer (ESO) theory, and fuzzy control (FC). The SMC is insensitive to both internal parameter uncertainties and external disturbances. The motivation for using ESO is to estimate the disturbance in real-time. The fuzzy parameter self-tuning strategy is proposed to adjust the switching gain on line according to the running state of the system. The stability of the system is guaranteed in the sense of the Lyapunov stability theorem. The effectiveness and robustness of the designed FSMC-ESO are illustrated by simulations.
\end{abstract}

\section{Introduction}

The 3-DOF serial-parallel hybrid humanoid arm (HHA) [1, 2] is composed of a 2-DOF parallel mechanism and a 1DOF serial mechanism, as shown in Figure 1, can be used in humanoid robots or automated production lines. Because of the special structure, the HHA combines the characteristics of series and parallel robot and is discovered in high speed and high positioning accuracy. The special structure introduces complexity in kinematics, dynamic equations, and coupling of the system and places greater demands on control methods.

Since HHA is a very complicated multiple-input multipleoutput (MIMO) nonlinear system with time-varying, strongcoupling characteristics, the design of robust controllers which is suitable for real-time control of HHA is one of the most challenging tasks, especially when HHA within inertia uncertainty and external disturbance. Advanced controller of robotic is a hot field in robotic research in recent years. Various advanced control strategies, either model-based control or model-free control, have been researched to improve the motion performance of the robotics [3-9]. The current trend of control approaches focuses on integrating conventional control techniques (e.g., adaptive control $[6,7]$ and slidingmode control [3-6]) with intelligent schemes (e.g., fuzzy theory $[7,8]$ and neural network $[5])$ in order to improve the performance of classical controllers.

About the control theory, the sliding-mode control is a useful and effective control scheme and is an efficient method to deal with uncertainties, time varying properties, nonlinearities, and bounded externals disturbances [3-5]. However, traditional SMC always has an undesired chattering phenomenon, which will inspire high-frequency unmodeled dynamics of the system and will eventually cause the loss of stability of system. Many methods are proposed to solve the chatting problem, like using saturation function instead of sign function [6], using fuzzy logic control $[7,8]$, and using new Lyapunov function having no discontinuous mathematical expressions [9].

Extended state observer (ESO) which is rooted in the active disturbance rejection control [10] regards all the unknown factors as disturbance and can observe and compensate the disturbance. In order to suppress the disturbance with removing its impact on the choice of the switching gain of sliding-mode control, ESO can be used here to make the disturbance estimated and compensated in the control 


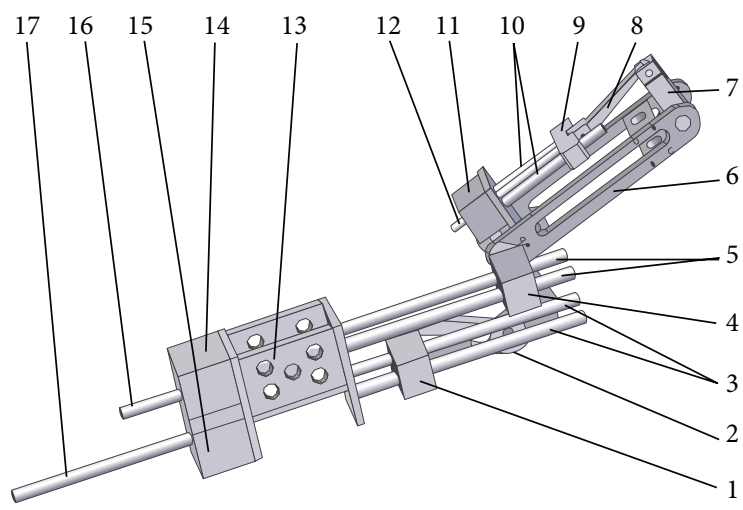

FIGURE 1: The prototype of 3-DOF forearm.

input, which implies a smaller switching gain and chattering phenomenon. The original concept of ESO is proposed for the nonlinear structure by Han [11-13] and further simplified and parameterized by Gao [14] Tian and Gao [15], which significantly simplifies the tuning of ESO parameters and makes ESO more suitable for practical applications $[16,17]$.

With the continuous development of the fuzzy logic control theory, its application on robots received more and more attentions [7-9]. The fuzzy logic can be used to improve the transient process of the robot system, which means the short transient time and small overshoot [18-20]. The knowledge about system characteristics coming from experts can be expressed by fuzzy logic rules, which makes this control method practical and simple for engineers.

In this paper, new design methods are developed that combine the advantages of sliding-mode control and ESO technique; the disturbance can be estimated by ESO and compensated by control law. To enhance the dynamic performance of HHA system, fuzzy control strategy is proposed to adjust the switching gain on line according to the running state of the system [20]. This control algorithm can be applied to manipulator systems with unmodeled dynamics, unstructured uncertainties, decoupling, and external disturbance.

The remainder of the paper is organized as follows. In Section 2, the structure of 3-DOF HHA is analyzed and the mathematical modeling of HHA is presented. In Section 3, the SMC, SMC-ESO, and FSMC-ESO for the HHA are proposed. Computer simulation results of the proposed SMC, SMC-ESO, and FSMC-ESO for the HHA are given in Section 4. Finally, Section 5 concludes the paper.

\section{3-DOF Serial-Parallel Hybrid Humanoid Arm}

2.1. The Structure of 3-DOF HHA. This 3-DOF serial-parallel hybrid humanoid arm is composed of a 2-DOF parallel mechanism which forms the upper arm of HHA and a 1-DOF serial mechanism which forms the forearm of HHA.

As shown in Figure 1, the 2-DOF parallel mechanism is composed of linear motors 14 and 15 and their movers 16 and 17, upper arm orbits 3 and 5, upper arm slip tubes 1

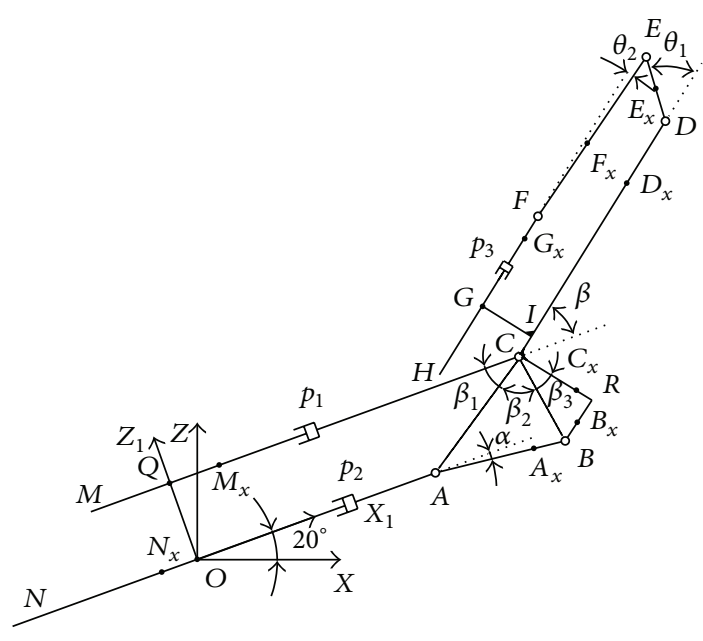

Figure 2: Structure of forearm in $\left\{O_{0}\right\}$ and $\left\{O_{1}\right\}$.

and 4, elbow handspike 2, and upper arm pedestal 13 . The 1DOF serial mechanism is composed of linear motor 11 and its mover 12 , forearm pedestal 6 , wrist handspike 8 , wrist 7 , forearm orbit 10, and forearm slip tube 9 . The motors 14 and 15 and upper arm orbit are fixed on upper arm pedestal. The motor 11 is fixed on forearm pedestal. The movers P1, P2, and P3 move forwards or backwards relatively to its main-body motors M1, M2, and M3, and the movement of motors drives the other components to achieve the desired action through the rotation axes.

The coordinates $\left\{\mathrm{O}_{0}\right\}$ and $\left\{\mathrm{O}_{1}\right\}$ are shown in Figure 2. $\left\{\mathrm{O}_{0}\right\}$ is the fixed reference coordinate system and $\left\{\mathrm{O}_{1}\right\}$ is attached to the upper arm of HHA. Referenced to the horizontal position, the upward inclination of the upper arm is up about $20^{\circ}$. Under the assumption of the regular shape, smooth surface and linear density steady of every component, the sketch of HHA in $\left\{\mathrm{O}_{0}\right\}$ and $\left\{\mathrm{O}_{1}\right\}$ is shown in Figure 2. The dot $Q, O, G$ is the center of mass of the motors M1, M2, and M3 and their pedestals, respectively. The $\operatorname{dot} C, A, F$ is the center of mass of the slip tubes S1, S2, and S3, respectively. The lengths of $A N$ and $C M$ are both $l_{1}$, and the lengths of $O Q, A B, R C, R B, C D, B C, E F, D E, I D, F H$ are $l_{0}, l_{2}, l_{3}, l_{4}, l_{5}$, $l_{6}, l_{11}, l_{10}, l_{15}$, and $l_{30}$, respectively. The lengths of $Q C, O A$, and $G F$ are defined as $q_{1}, q_{2}$, and $q_{3}$, respectively.

2.2. Dynamic Modeling for HHA. Using the Lagrange approach, the dynamic model of the HHA [4] can be written as

$$
\mathbf{M}(\mathbf{q}) \ddot{\mathbf{q}}+\mathbf{C}(\mathbf{q}, \dot{\mathbf{q}}) \dot{\mathbf{q}}+\mathbf{G}(\mathbf{q})+\tau_{d}=\boldsymbol{\tau},
$$

where $\mathbf{q}, \dot{\mathbf{q}}$, and $\ddot{\mathbf{q}} \in R^{3}$ are the vectors of slip tubes position, velocity, and acceleration, respectively. $\mathbf{M}(\mathbf{q})=\mathbf{M}_{0}(\mathbf{q})+$ $\Delta \mathbf{M}(\mathbf{q}) \in R^{3 \times 3}$ is the inertia matrix, $\mathbf{C}(\mathbf{q}, \dot{\mathbf{q}})=\mathbf{C}_{0}(\mathbf{q}, \dot{\mathbf{q}})+$ $\Delta \mathbf{C}(\mathbf{q}, \dot{\mathbf{q}}) \in R^{3 \times 3}$ is the centripetal and Coriolis terms, $\mathbf{G}(\mathbf{q})=$ $\mathbf{G}_{0}(\mathbf{q})+\Delta \mathbf{G}(\mathbf{q}) \in R^{3}$ is the vector of gravitational force, $\boldsymbol{\tau}_{d} \in R^{3}$ is the vector of generalized input due to external disturbance with $\left\|\tau_{d}\right\| \leq d$, and $\tau \in R^{3}$ is the input force vector. Here, $\mathbf{M}_{0}(\mathbf{q}), \mathbf{C}_{0}(\mathbf{q}, \dot{\mathbf{q}})$, and $\mathbf{G}_{0}(\mathbf{q})$ are the nominal 
parts calculated by Lagrange method, where $\mathbf{M}_{0}(\mathbf{q})$ is a symmetric positive definite matrix. $\Delta \mathbf{M}_{0}(\mathbf{q}), \Delta \mathbf{C}_{0}(\mathbf{q}, \dot{\mathbf{q}})$, and $\Delta \mathbf{G}_{0}(\mathbf{q})$ represent the perturbations in the system matrices. Then the dynamic model of HHA can be rewritten as

$$
\mathbf{M}_{0}(\mathbf{q}) \ddot{\mathbf{q}}+\mathbf{C}_{0}(\mathbf{q}, \dot{\mathbf{q}}) \dot{\mathbf{q}}+\mathbf{G}_{0}(\mathbf{q})+\tau_{d}+\mathbf{F}(\mathbf{q}, \dot{\mathbf{q}}, \ddot{\mathbf{q}})=\tau,
$$

where

$$
\mathbf{F}(\mathbf{q}, \dot{\mathbf{q}}, \ddot{\mathbf{q}})=\Delta \mathbf{M}_{0}(\mathbf{q}) \ddot{\mathbf{q}}+\Delta \mathbf{C}_{0}(\mathbf{q}, \dot{\mathbf{q}}) \dot{\mathbf{q}}+\Delta \mathbf{G}_{0}(\mathbf{q}) \in R^{3}
$$

is the system uncertainty and satisfies $\|\mathbf{F}(\mathbf{q}, \dot{\mathbf{q}}, \ddot{\mathbf{q}})\| \leq b_{0}+$ $b_{1}\|\mathbf{q}\|+b_{2}\|\dot{\mathbf{q}}\|^{2}[5,21]$.

\section{ESO-Based Self-Tuning Sliding-Mode Control (FSMC-ESO) for HHA}

In this section, to ensure that the motion of the HHA can follow the desired trajectory, three dynamic controllers are introduced. First, we design a conventional sliding-mode controller (SMC) for the HHA in the thrust level. Second, the SMC-ESO is proposed for solving the chatting problem. Finally, the FSMC-ESO is proposed to enhance the dynamic performance and further solve the chattering problem of HHA system. The convergence of the complete equations of motion of the FSMC-ESO based HHA is confirmed by the Lyapunov stability theorem.

3.1. SMC Design. This section describes the SMC that is applied to the HHA. Sliding-mode control (SMC) is one of the effective nonlinear robust control approaches since it provides system dynamics with an invariance property to uncertainties once the system dynamics are controlled in the sliding mode. The SMC design can be decoupled in two steps. The first step is the selection of an appropriate sliding surface. The second step, an approaching law, is designed so that it will drive the system state toward sliding surface and guarantee the stability of the system.

The realistic model (2) can be reformulated as

$$
\ddot{\mathbf{q}}=-\mathbf{M}_{0}{ }^{-1} \mathbf{C}_{0} \dot{\mathbf{q}}-\mathbf{M}_{0}^{-1} \mathbf{G}_{0}-\mathbf{d}(t)+\mathbf{M}_{0}^{-1} \boldsymbol{\tau},
$$

where $\mathbf{d}(t)=\mathbf{M}_{0}^{-1}\left(\boldsymbol{\tau}_{d}+\mathbf{F}\right)$.

Assuming $\overline{\mathbf{d}}(t)$ is the upper bound of $\mathbf{d}(t)$, then we can get $\mathbf{d}(t) \leq|\mathbf{d}(t)| \leq \overline{\mathbf{d}}(t)$.

For 3-DOF HHA, define the tracking error:

$$
\mathbf{e}=\mathbf{q}_{d}-\mathbf{q}
$$

where $\mathbf{q}_{d}$ is the desired position trajectory of slip tubes.

The sliding surface in the space of tracking error is defined as

$$
\mathbf{S}=\dot{\mathbf{e}}+\mathbf{K e},
$$

where $\mathbf{S}=\left[S_{1}, S_{2}, S_{3}\right]^{T}, \mathbf{K}=\operatorname{diag}\left[K_{1}, K_{2}, K_{3}\right]$, and $K_{1}, K_{2}$, $K_{3}>0$.

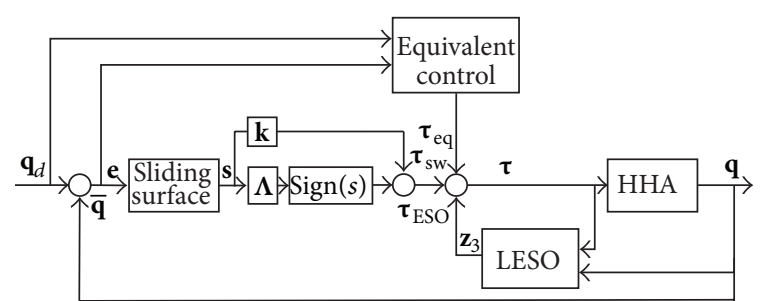

FIgURE 3: The block diagram of the SMC-ESO.

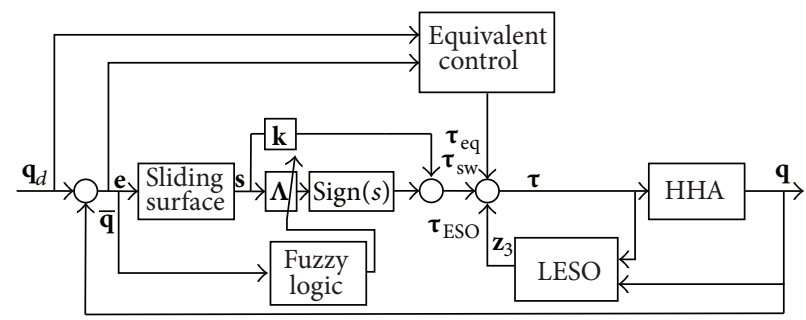

FIgURE 4: The block diagram of the FSMC-ESO.

Select the approaching law as

$$
\dot{\mathbf{S}}=-\boldsymbol{\Lambda} \operatorname{sgn}(\mathbf{S})-\mathbf{k S},
$$

where $\boldsymbol{\Lambda}=\operatorname{diag}\left[\lambda_{1}, \lambda_{2}, \lambda_{3}\right], \mathbf{k}=\operatorname{diag}\left[k_{1}, k_{2}, k_{3}\right]$, and $\lambda_{1}, \lambda_{2}$, $\lambda_{3}, k_{1}, k_{2}, k_{3}>0$.

For the 3-DOF HHA system (4), the sliding-mode control law is design as

$$
\begin{gathered}
\boldsymbol{\tau}=\boldsymbol{\tau}_{\mathrm{eq}}+\boldsymbol{\tau}_{\mathrm{sw}}, \\
\boldsymbol{\tau}_{\mathrm{eq}}=\mathbf{M}_{0} \ddot{\mathbf{q}}_{d}+\mathbf{M}_{0} \mathbf{K} \dot{\mathbf{e}}+\mathbf{C}_{0} \dot{\mathbf{q}}+\mathbf{G}, \\
\boldsymbol{\tau}_{\mathrm{sw}}=\mathbf{M}_{0} \boldsymbol{\Lambda} \operatorname{sgn}(\mathbf{S})+\mathbf{M}_{0} \mathbf{k S},
\end{gathered}
$$

where, $\boldsymbol{\tau}_{\text {eq }}$ is equivalent control, that can make the system state remain on the sliding surface with no disturbance and $\boldsymbol{\tau}_{\mathrm{sw}}$ is switching control, that can drive the system state to the sliding surface.

If $\boldsymbol{\Lambda}$ is chosen as

$$
\Lambda \geq \overline{\mathbf{d}}(t)
$$

the tracking error e will asymptotically converge to zero.

However, the upper bound of uncertainties, which is required in the conventional SMC system, is difficult to obtain precisely in advance for practical applications. If the switching gains are selected too large, the sign function will result in serious chattering phenomena in the control efforts. The undesired chattering control efforts will wear the bearing mechanism and might excite unstable system dynamics. Hence, the ESO can be adopted here to make the total disturbance estimated and compensated in the control input, which implies the decrease of the chattering and control power.

3.2. ESO-Based SMC Design. The ESO views the system model uncertainties and external disturbances as 
TABLE 1: Fuzzy associative memory (FAM) table for switching gain $\lambda_{i}$.

\begin{tabular}{|c|c|c|c|c|c|c|c|}
\hline \multirow{2}{*}{$e_{i}$} & \multicolumn{7}{|c|}{$\dot{e}_{i}$} \\
\hline & NB & NM & NS & $\mathrm{ZO}$ & PS & $\mathrm{PM}$ & PB \\
\hline NB & $\mathrm{M}$ & $\mathrm{B}$ & VB & VVB & $\mathrm{VB}$ & B & $\mathrm{M}$ \\
\hline NM & $S$ & $\mathrm{M}$ & B & VB & B & $\mathrm{M}$ & $S$ \\
\hline NS & VS & $S$ & $\mathrm{M}$ & B & $\mathrm{M}$ & $S$ & VS \\
\hline $\mathrm{ZO}$ & VVS & VS & $S$ & $\mathrm{M}$ & $S$ & VS & VVS \\
\hline PS & VS & $S$ & $\mathrm{M}$ & B & $\mathrm{M}$ & $S$ & VS \\
\hline PM & $S$ & M & B & VB & B & $\mathrm{M}$ & $S$ \\
\hline PB & M & B & VB & VVB & VB & B & M \\
\hline
\end{tabular}

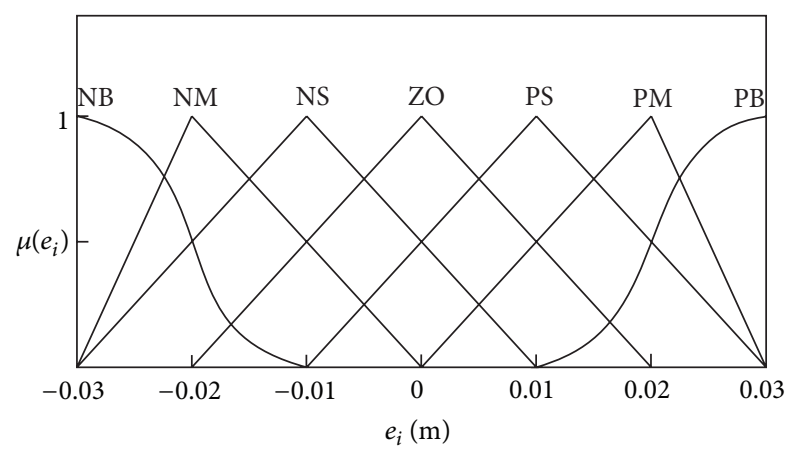

(a)

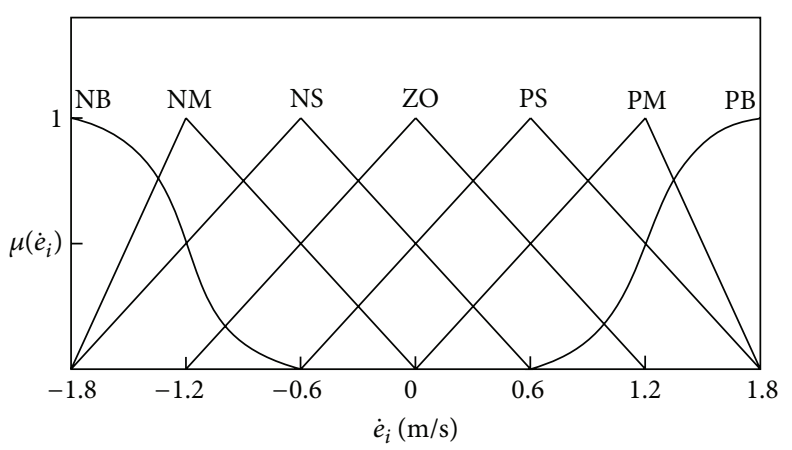

(b)

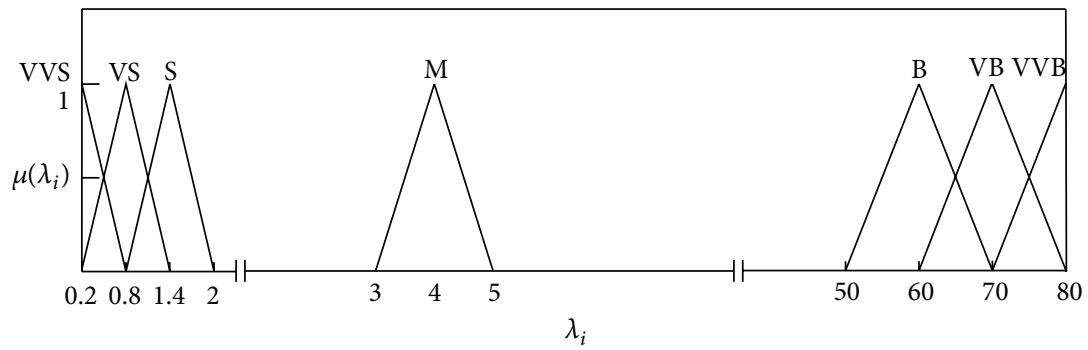

(c)

FIgURE 5: Membership functions (a) error, (b) derivative of error, and (c) switching gain.

the extended state to be estimated. Here, the ESO can be designed for estimating the total disturbances $\mathbf{d}(t)$ existing in system (4). The block diagram of the SMC-ESO is shown in Figure 3.

Let $\mathbf{D}(t)=-\mathbf{d}(t)$, assuming $\mathbf{D}(t)$ is differentiable and $\dot{\mathbf{D}}(t)=\mathbf{h}(t)$. Let $\mathbf{x}_{1}=\mathbf{q}, \mathbf{x}_{2}=\dot{\mathbf{q}}, \mathbf{x}_{3}=\mathbf{D}(t)$, the augmented model of (4) is

$$
\begin{gathered}
\dot{\mathbf{x}}_{1}=\mathbf{x}_{2}, \\
\dot{\mathbf{x}}_{2}=\mathbf{x}_{3}-\mathbf{M}_{0}{ }^{-1} \mathbf{C}_{0} \mathbf{x}_{2}-\mathbf{M}_{0}{ }^{-1} \mathbf{G}_{0}+\mathbf{M}_{0}{ }^{-1} \boldsymbol{\tau}, \\
\dot{\mathbf{x}}_{3}=\mathbf{h}(t) .
\end{gathered}
$$

The ESO of system (11) can be designed as

$$
\begin{gathered}
\dot{\mathbf{z}}_{1}=\mathbf{z}_{2}+\boldsymbol{\beta}_{1} \mathrm{E}_{1}, \\
\dot{\mathbf{z}}_{2}=\mathbf{z}_{3}+\boldsymbol{\beta}_{2} \mathrm{E}_{1}-\mathbf{M}_{0}^{-1} \mathbf{C}_{0} \mathbf{x}_{2}-\mathbf{M}_{0}{ }^{-1} \mathbf{G}_{0}+\mathbf{M}_{0}{ }^{-1} \boldsymbol{\tau}, \\
\dot{\mathbf{z}}_{3}=\boldsymbol{\beta}_{3} \mathbf{E}_{1},
\end{gathered}
$$

where $\mathbf{E}_{1}$ is the estimation error of the ESO, $\mathbf{Z}_{1}, \mathbf{Z}_{2}$, and $\mathbf{Z}_{3}$ are the observer outputs, and $\boldsymbol{\beta}_{1}=\operatorname{diag}\left[\beta_{11}, \beta_{12}, \beta_{13}\right]$, $\boldsymbol{\beta}_{2}=\operatorname{diag}\left[\beta_{21}, \beta_{22}, \beta_{23}\right]$, and $\boldsymbol{\beta}_{3}=\operatorname{diag}\left[\beta_{31}, \beta_{32}, \beta_{33}\right]$ are the observer gains. With the disturbances $\mathbf{D}(t)$ estimated by the ESO, the control law (8) is modified as

$$
\begin{gathered}
\boldsymbol{\tau}=\boldsymbol{\tau}_{\mathrm{eq}}+\boldsymbol{\tau}_{\mathrm{sw}}+\boldsymbol{\tau}_{\mathrm{ESO}}, \\
\boldsymbol{\tau}_{\mathrm{ESO}}=-\mathbf{M}_{0} \mathbf{z}_{3} .
\end{gathered}
$$

Theorem 1. For the 3-DOF HHA (4), if the sliding surface is chosen as (6), the reaching law is chosen as (7) and the control law is designed as (13), there exist observer gains $\boldsymbol{\beta}_{1}, \boldsymbol{\beta}_{2}$, and $\boldsymbol{\beta}_{3}$ and switching gains $\boldsymbol{\Lambda}$ such that the tracking error $\mathbf{e}$ and the estimation error $\mathbf{E}_{1}$ asymptotically converge to zero.

Proof. In order to examine the stability of the closed loop system, the stability of the ESO is demonstrated firstly. Let the estimation errors of ESO $\mathbf{E}_{1}=\mathbf{x}_{1}-\mathbf{z}_{1}, \mathbf{E}_{2}=\mathbf{x}_{2}-\mathbf{z}_{2}$, and 


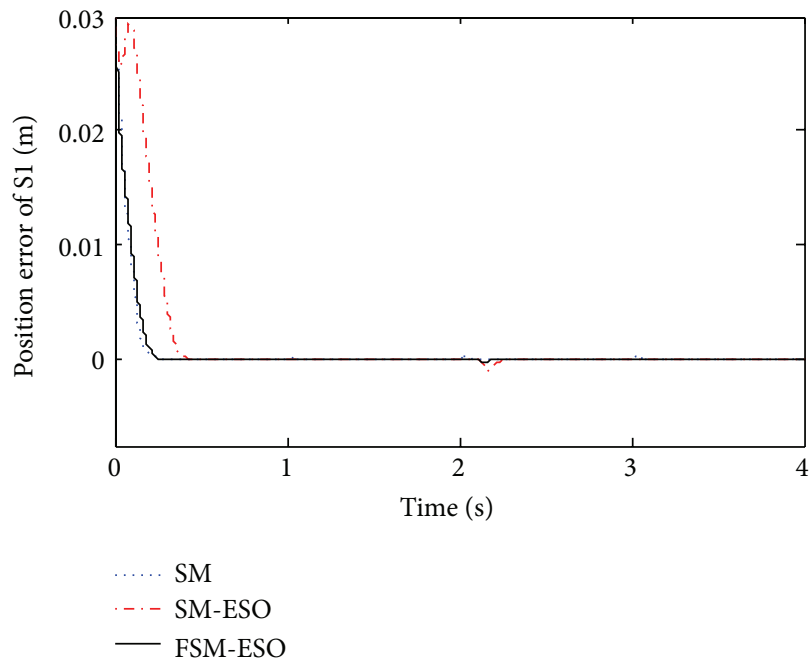

(a)

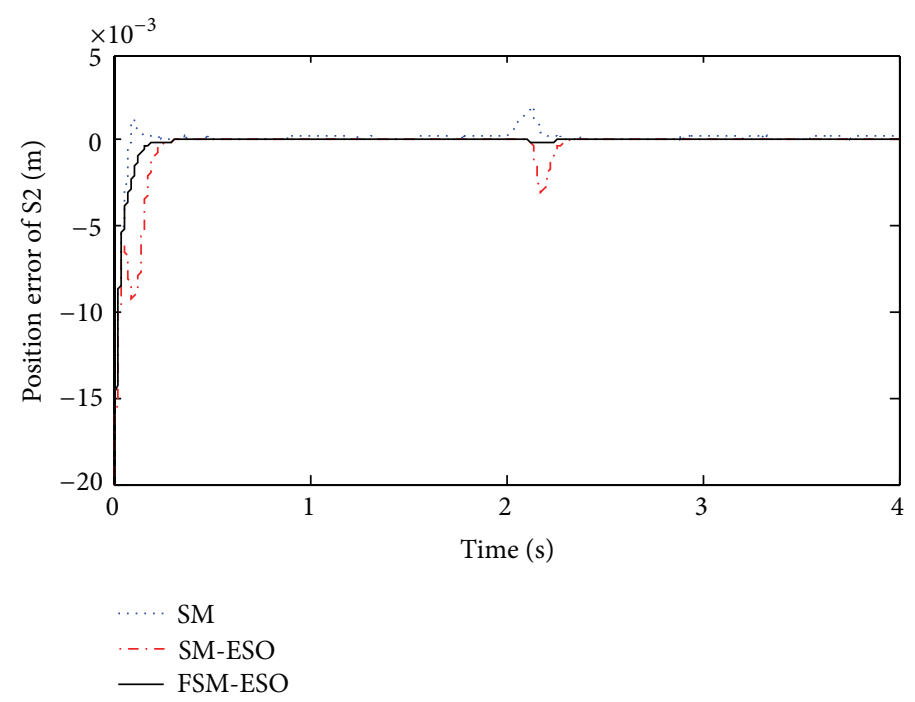

(b)

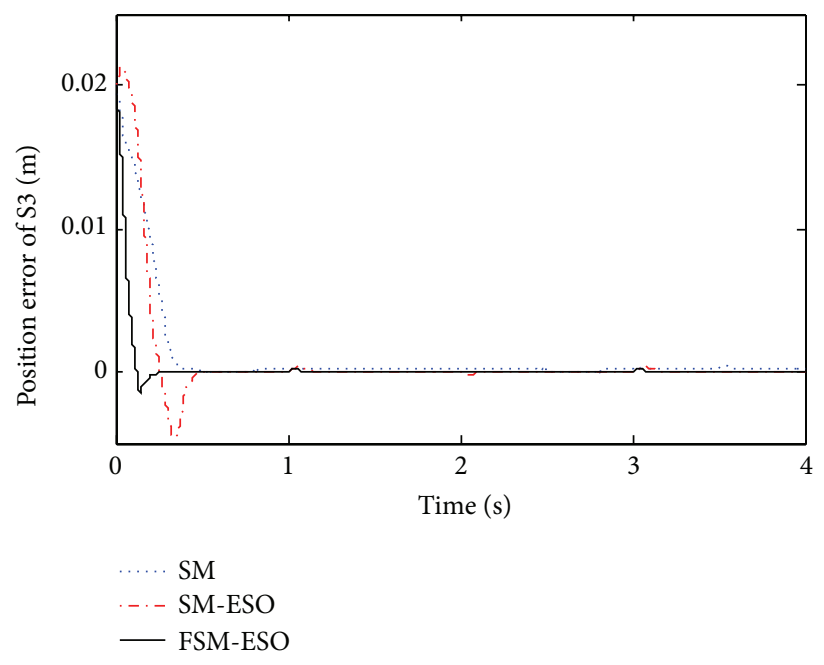

(c)

Figure 6: (a) Position tracking error of S1, (b) position tracking error of S2, and (c) position tracking error of S3.

$\mathbf{E}_{3}=\mathbf{x}_{3}-\mathbf{z}_{3}$, then the observer error dynamics are expressed as

$$
\begin{gathered}
\dot{\mathbf{E}}_{1}=\mathbf{E}_{2}-\boldsymbol{\beta}_{1} \mathbf{E}_{1}, \\
\dot{\mathbf{E}}_{2}=\mathbf{E}_{3}-\boldsymbol{\beta}_{2} \mathbf{E}_{1}, \\
\dot{\mathbf{E}}_{3}=\mathbf{h}(t)-\boldsymbol{\beta}_{3} \mathbf{E}_{1} .
\end{gathered}
$$

By choosing appropriate observer gains $\boldsymbol{\beta}_{1}, \boldsymbol{\beta}_{2}$, and $\boldsymbol{\beta}_{3}$, the stability of the ESO can be guaranteed and illustrated in [16]. As the observer is stable, the derivative of errors $\dot{\mathbf{E}}_{1}=0$, $\dot{\mathbf{E}}_{2}=0$, and $\dot{\mathbf{E}}_{3}=0$, then the errors of estimation can be written as

$$
\mathbf{E}_{1}=\frac{\mathbf{h}(t)}{\beta_{1}}
$$

$$
\begin{aligned}
& \mathbf{E}_{2}=\boldsymbol{\beta}_{1} \frac{\mathbf{h}(t)}{\boldsymbol{\beta}_{2}}, \\
& \mathbf{E}_{3}=\boldsymbol{\beta}_{2} \frac{\mathbf{h}(t)}{\boldsymbol{\beta}_{3}} .
\end{aligned}
$$

For the 3-DOF serial-parallel hybrid humanoid arm, $\overline{\mathbf{d}}(t)$ is the upper bound of $\mathbf{d}(t)$, even if the $\mathbf{d}(t)$ may contain large uncertainties, the observation error of the ESO can be down to the small enough by adjust the parameters $\boldsymbol{\beta}_{1}, \boldsymbol{\beta}_{2}$, and $\boldsymbol{\beta}_{3}$. Thus, via tuning these parameters properly, the estimation errors $\mathbf{E}_{1}, \mathbf{E}_{2}$, and $\mathbf{E}_{3}$ can be limited to be small enough.

Having shown that the observer errors converge into the residual set of zero, it remains to show that the closed loop 


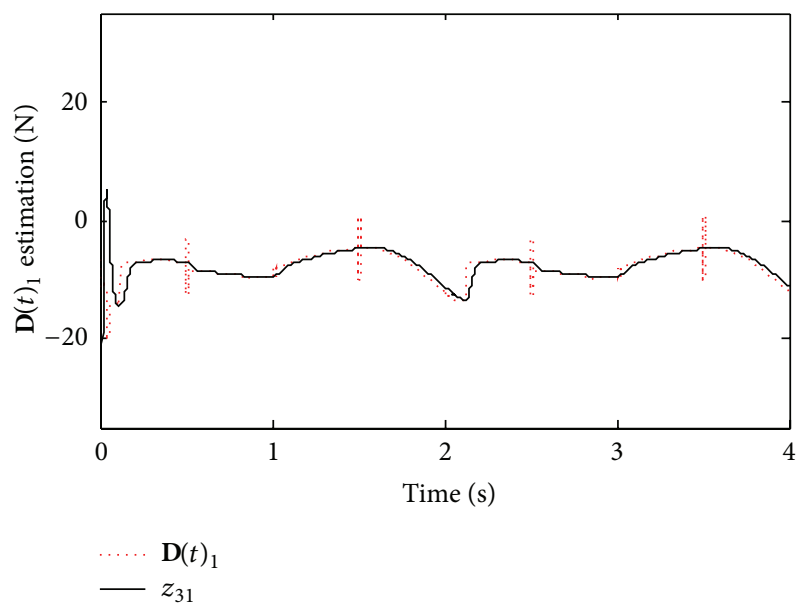

(a)

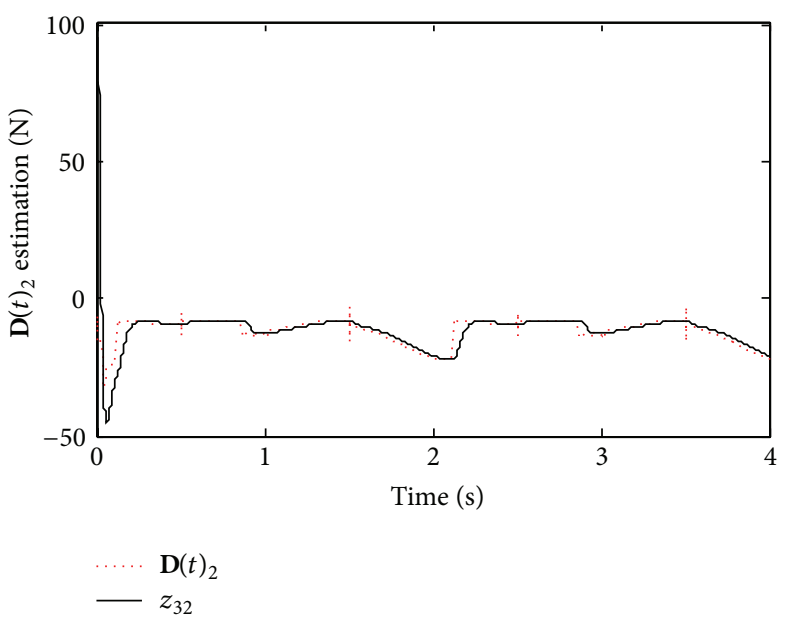

(b)

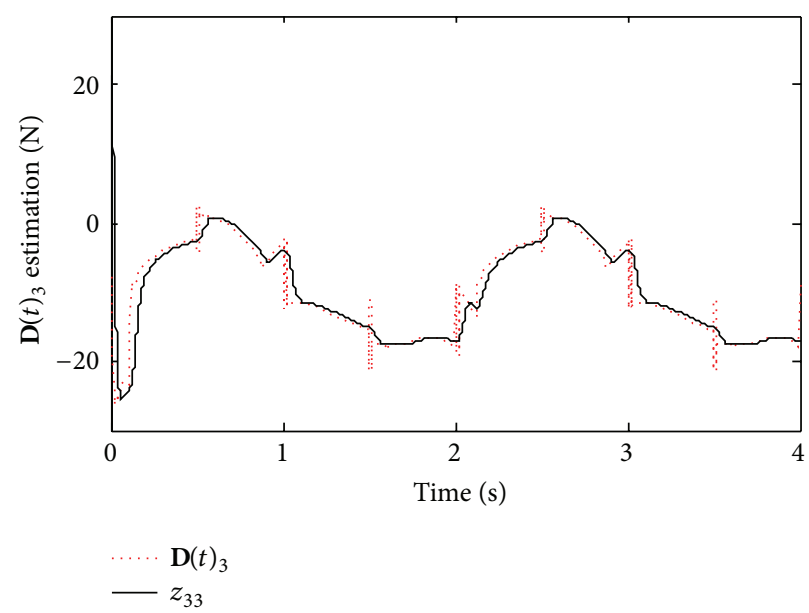

(c)

Figure 7: (a) Disturbance estimation of $\mathbf{D}(t)_{1}$, (b) disturbance estimation of $\mathbf{D}(t)_{2}$, and (c) disturbance estimation of $\mathbf{D}(t)_{3}$.

errors converge to zero. Considering the Lyapunov function candidate with the sliding surface given by (6), we obtain

$$
\begin{aligned}
V & =\frac{1}{2} \mathbf{S}^{T} \mathbf{S} \\
\dot{V} & =\mathbf{S}^{T} \dot{\mathbf{S}} \\
= & \mathbf{S}^{T}(\ddot{\mathbf{e}}+\mathbf{K} \dot{\mathbf{e}})=\mathbf{S}^{T}\left(\ddot{\mathbf{q}}_{d}-\ddot{\mathbf{q}}+\mathbf{K} \dot{\mathbf{e}}\right) \\
= & \mathbf{S}^{T}\left(\ddot{\mathbf{q}}_{d}+\mathbf{M}_{0}{ }^{-1} \mathbf{C}_{0} \dot{\mathbf{q}}+\mathbf{M}_{0}{ }^{-1} \mathbf{G}_{0}+\mathbf{d}(t)\right. \\
& \left.\quad-\mathbf{M}_{0}{ }^{-1} \boldsymbol{\tau}+\mathbf{K} \dot{\mathbf{e}}\right) .
\end{aligned}
$$

Combine (13) and (17), we get

$$
\begin{aligned}
\dot{V}=\mathbf{S}^{T}\left\{\ddot{\mathbf{q}}_{d}+\mathbf{M}_{0}^{-1} \mathbf{C}_{0} \dot{\mathbf{q}}+\mathbf{M}_{0}^{-1} \mathbf{G}_{0}+\mathbf{d}(t)+\mathbf{K} \dot{\mathbf{e}}\right. \\
-\mathbf{M}_{0}^{-1}\left[\mathbf{M}_{0} \ddot{\mathbf{q}}_{d}+\mathbf{M}_{0} \mathbf{K} \dot{\mathbf{e}}+\mathbf{C}_{0} \dot{\mathbf{q}}+\mathbf{G}\right. \\
\left.\left.+\mathbf{M}_{0} \boldsymbol{\Lambda} \operatorname{sgn}(\mathbf{S})+\mathbf{M}_{0} \mathbf{k S}-\mathbf{M}_{0} \mathbf{z}_{3}\right]\right\}
\end{aligned}
$$

$$
\begin{aligned}
& =\mathbf{S}^{T}\left\{\mathbf{d}(t)+\mathbf{z}_{3}-\boldsymbol{\Lambda} \operatorname{sgn}(\mathbf{S})-\mathbf{k} \mathbf{S}\right\} \\
& =\mathbf{S}^{T}\left\{\mathbf{E}_{3}-\boldsymbol{\Lambda} \operatorname{sgn}(\mathbf{S})-\mathbf{k} \mathbf{S}\right\} \\
& =\mathbf{S}^{T}\left\{\mathbf{E}_{3}-\boldsymbol{\Lambda} \operatorname{sgn}(\mathbf{S})\right\}-\mathbf{S}^{T} \mathbf{k S} .
\end{aligned}
$$

As we know $\mathbf{E}_{3}$ converges into the residual set of zero finally, (18) becomes

$$
\dot{V} \approx-\boldsymbol{\Lambda} \mathbf{S}^{T} \operatorname{sgn}(\mathbf{S})-\mathbf{S}^{T} \mathbf{k} \mathbf{S}
$$

Let $\boldsymbol{\Lambda} \geq 0, \mathbf{k} \geq 0$, we can conclude that $\dot{V} \leq 0$ is always satisfied. Thus the closed loop system is asymptotically stable and the error state trajectory converges to the sliding surface $\mathbf{S}(t)=0$. As the disturbance $\mathbf{D}(t)$ is estimated and compensated by ESO, the switching gain $\boldsymbol{\Lambda}$ can be selected in smaller values, which implies the decrease of the chattering and control power. 


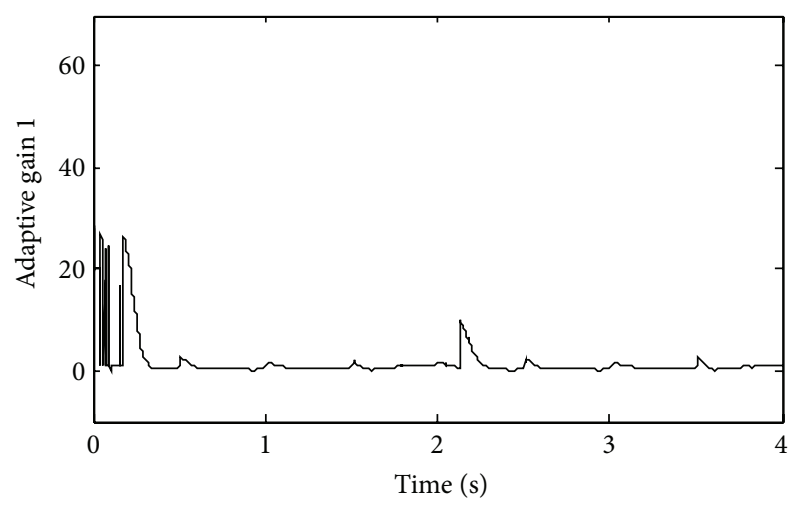

(a)

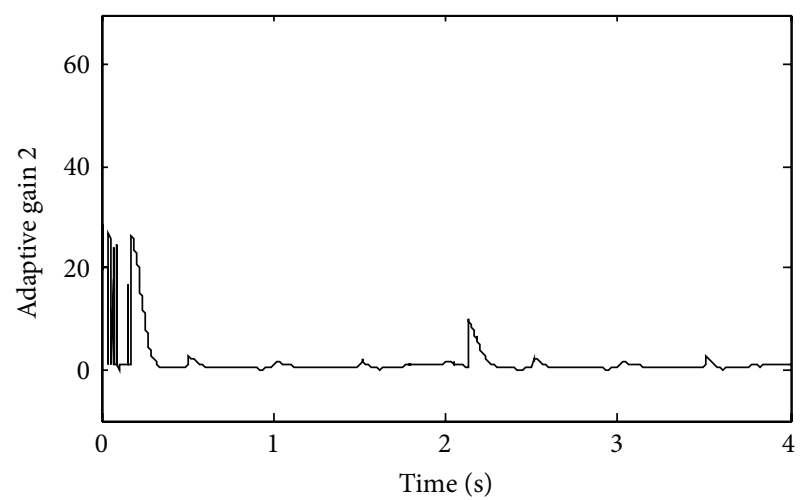

(b)

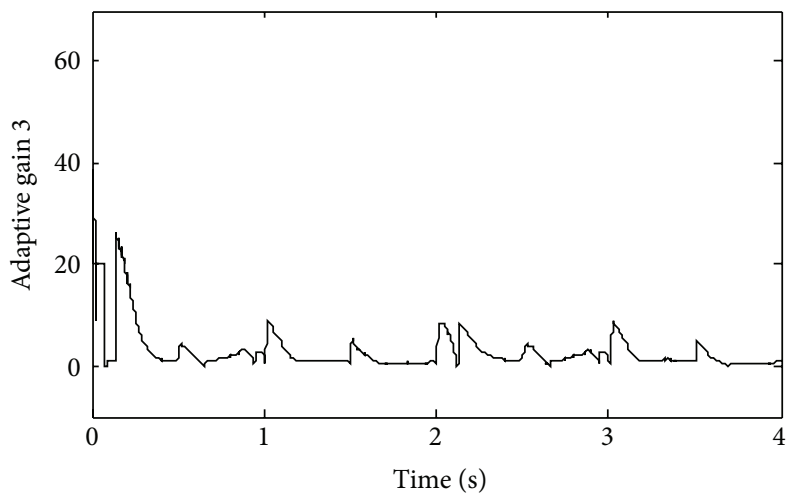

(c)

FIGURE 8: (a) The variety of self-turning gains $\lambda_{1}$, (b) the variety of self-turning gains $\lambda_{2}$, and (c) the variety of self-turning gains $\lambda_{3}$.

3.3. ESO-Based FSMC Design. In Section 3.2, with smaller values of switching gain $\Lambda$, the chatting problem is solved, but the dynamic performance of the system will be influenced by small gains of SMC-ESO. In this section, the ESO-based FSMC with varying switching gain is presented (Table 1). The switching gain is changed dynamically by the fuzzy logic unit in order to improve the performance of the controller. The general structure of the proposed FSMC-ESO is given in Figure 4.

As shown in Figure 5, the membership functions are used for the fuzzification of the inputs, which are error $e_{i}$ and derivative of error $\dot{e}_{i}$. Here NB, NM, NS, ZO, PS, PM, and $\mathrm{PB}$ stand for negative big, negative medium, negative small, zero, positive small, positive medium, and positive big, respectively. Also VVS, VS, S, M, B, VB, and VVB stand for very very small, very small, small, medium, big, very big, and very very big.

For output, the membership functions are presented in Figure 5(c). The definition of the membership functions gives the ability to make the control gain value of the proposed controller smaller or bigger than the gain of the conventional sliding-mode controller whenever necessary.

Since the control gain $\Lambda \geq 0$ is always satisfied during the fuzzy adaptation, so if we select $\mathbf{k} \geq 0$, one can conclude that $\dot{V} \leq 0$ is always satisfied and the Lyapunov stability of the controlled system is preserved. Thus the closed loop system is asymptotically stable and tracking error will converge to zero finally. The positive definiteness of the control gain is guaranteed by defining the membership functions within the positive universe of discourse.

\section{Simulation}

A set of numerical simulations is used here to verify the effectiveness of the proposed SMC, SMC-ESO, and FSMCESO. The numerical values of parameters of the HHA are listed as follows: $l_{0}=0.5 \mathrm{~m}, l_{1}=0.09 \mathrm{~m}, l_{2}=0.15 \mathrm{~m}$, $l_{3}=0.09 \mathrm{~m}, l_{4}=0.05 \mathrm{~m}, l_{6}=0.103 \mathrm{~m}, l_{10}=0.065 \mathrm{~m}, l_{11}=$ $0.19 \mathrm{~m}, l_{15}=0.28 \mathrm{~m}, l_{30}=0.19 \mathrm{~m}, \rho_{2}=1.5 \mathrm{~kg} / \mathrm{m}, \rho_{3}=$ $2 \mathrm{~kg} / \mathrm{m}, \rho_{4}=1 \mathrm{~kg} / \mathrm{m}, m_{9}=0.5 \mathrm{~kg}$, and $g=9.8 \mathrm{~m} / \mathrm{s}^{2}$.

Normally, the distributed noise and the parameter uncertainties of the system always exist, which can be thought as disturbing thrust acting on the slip tubes, and are assumed to be time-varying as

$$
\mathbf{F}+\boldsymbol{\tau}_{d}=\left[\begin{array}{c}
10+20 \dot{q}_{1}+20 \sin \left(q_{1}\right)+5 \operatorname{sgn}\left(\dot{q}_{1}\right) \\
10+20 \dot{q}_{2}+20 \sin \left(q_{2}\right)+5 \operatorname{sgn}\left(\dot{q}_{2}\right) \\
8+15 \dot{q}_{3}+15 \sin \left(q_{3}\right)+3 \operatorname{sgn}\left(\dot{q}_{3}\right)
\end{array}\right] .
$$

The desired trajectory used here is given as $q_{d 1}=$ $0.25-0.05 \sin (\pi t), q_{d 2}=0.15+0.05 \sin (\pi t)$, and $q_{d 3}=$ $0.09+0.03 \cos (\pi t)$. The parameters of the sliding surface 


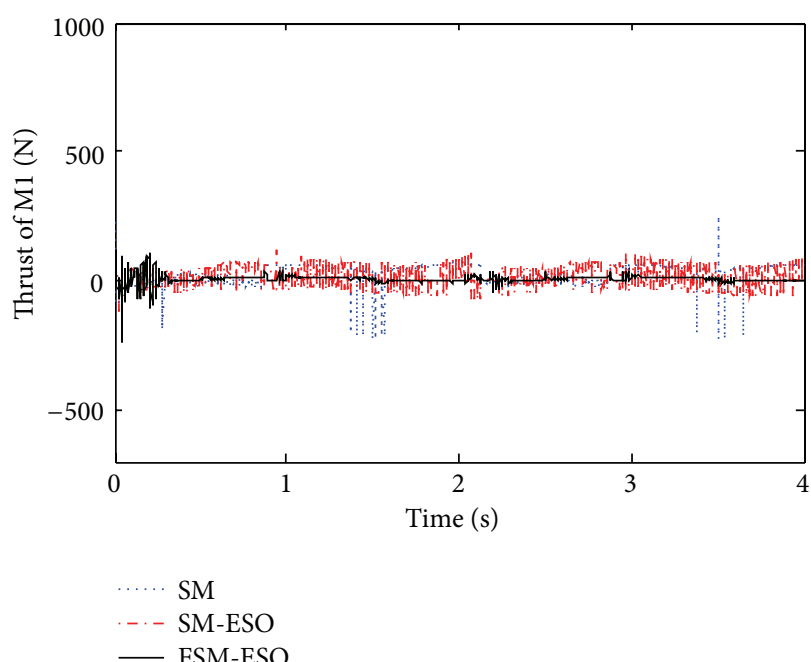

(a)

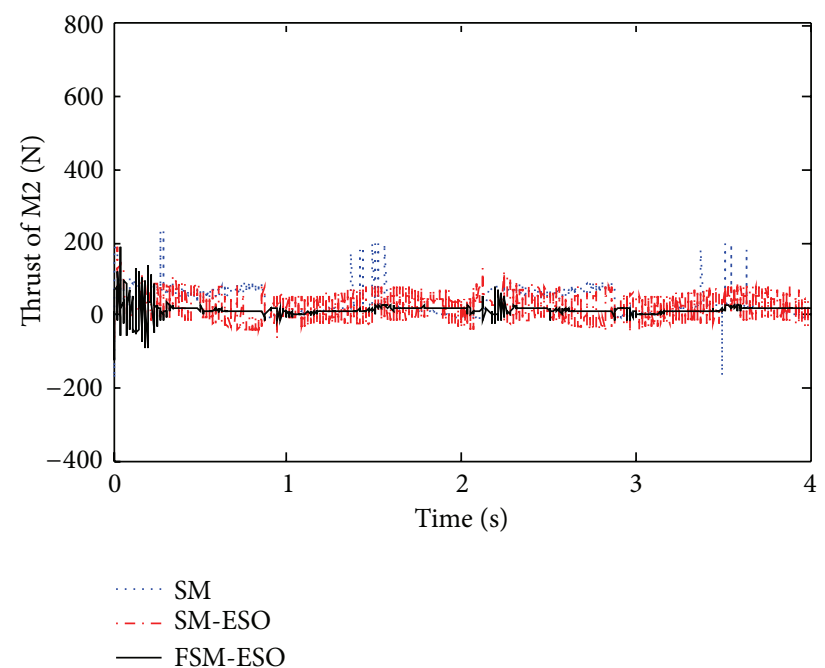

(b)

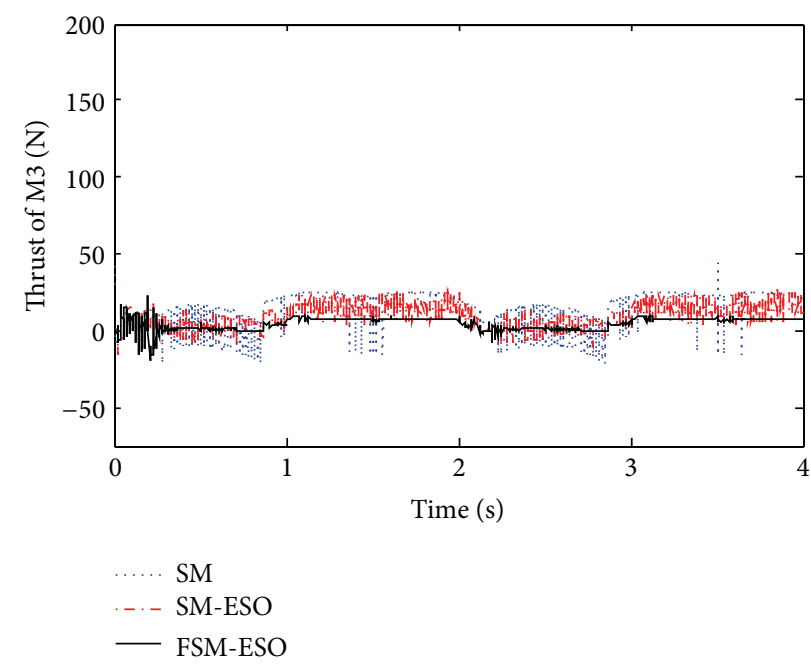

(c)

Figure 9: (a) Input thrust of M1, (b) input thrust of M2, and (c) input thrust of M3.

are $\mathbf{K}=\operatorname{diag}(30,30,30)$. For the conventional SMC, $\boldsymbol{\Lambda}=$ $\operatorname{diag}(16,21,18)$ and $\mathbf{k}=\operatorname{diag}(5,5,5)$. For SMC-ESO, $\boldsymbol{\Lambda}=$ $\operatorname{diag}(3,7,4)$ and $\mathbf{k}=\operatorname{diag}(5,5,5)$. For FSMC-ESO, $\boldsymbol{\Lambda}$ is adjusted by fuzzy $\operatorname{logic}, \mathbf{k}=\operatorname{diag}(5,5,5)$. For the ESO used in SMC-ESO and FSMC-ESO, $\left.\boldsymbol{\beta}_{1}=\operatorname{diag}(240,19200,512000)\right)$ and $\beta_{2}=\beta_{3}=\operatorname{diag}(180,10800,216000)$. Figure 6 shows the tracking position error of slip tubes 1, 2, and 3. Figure 7 shows the disturbance estimation of $D_{1}(t), D_{2}(t)$, and $D_{3}(t)$. Figure 8 shows the variety of self-turning gains $\lambda_{1}, \lambda_{2}$, and $\lambda_{3}$. Figure 9 shows the input thrust of $M_{1}, M_{2}$, and $M_{3}$.

From the simulation results, it is concluded that our proposed FSMC-ESO shows superior performance for each of the three slip tubes. Simulation results show that the proposed FSMC-ESO has smaller overshoot error values than both conventional SMC and SMC-ESO. From Figure 9 it is observed that both FSMC-ESO and SMC-ESO have smaller chatting problems in input thrust than the conventional method dealt with in this paper. It is observed that the proposed FSMC-ESO has the smallest chatting problem and best performance among the three controllers we proposed, which proves the efficiency of the proposed controller.

\section{Conclusion}

This present paper has described the development of ESObased self-tuning sliding-mode control (FSMC-ESO) where it has been successfully applied for the motion control of a serial-parallel hybrid humanoid arm (HHA) in the presence of uncertainties and disturbances. The ESO can estimate the disturbance in real-time and does not require the detailed information of uncertainty and disturbance, the ESO only needs the upper bound of the uncertainty and disturbance, and the switching gain is adjusted by the fuzzy logic on line according to the running state of the system. The FSMC-ESO, 
with the advantages of SMC, ESO, and parameter selftuning strategy, has shown a significant improvement over the conventional SMC under the same conditions. Meanwhile, the chattering phenomenon that frequently appears in the conventional SMC is also weakened by the fuzzy control. The effectiveness of the designed FSMC-ESO strategy was illustrated by simulation examples.

\section{Conflict of Interests}

The authors declare that there is no conflict of interests regarding the publication of this paper.

\section{References}

[1] Y. Wang, R. Shi, and H. Wang, "Dynamic modeling and fuzzy self-tuning disturbance decoupling control for a 3-DOF serial-parallel hybrid humanoid arm," Advances in Mechanical Engineering, vol. 2013, Article ID 286074, 14 pages, 2013.

[2] Y. Wang and Z. Jin, "Dynamics modeling and robust trajectory tracking control for a class of hybrid humanoid arm based on neural network," Chinese Journal of Mechanical Engineering, vol. 22, no. 3, pp. 355-363, 2009.

[3] S. Yu, X. Yu, B. Shirinzadeh, and Z. Man, "Continuous finitetime control for robotic manipulators with terminal sliding mode," Automatica, vol. 41, no. 11, pp. 1957-1964, 2005.

[4] M. B. R. Neila and D. Tarak, "Adaptive terminal sliding mode control for rigid robotic manipulators," International Journal of Automation and Computing, vol. 8, no. 2, pp. 215-220, 2011.

[5] T. Sun, H. Pei, Y. Pan, H. Zhou, and C. Zhang, "Neural networkbased sliding mode adaptive control for robot manipulators," Neurocomputing, vol. 74, no. 14-15, pp. 2377-2384, 2011.

[6] C. Chen, T. S. Li, Y. Yeh, and C. Chang, "Design and implementation of an adaptive sliding-mode dynamic controller for wheeled mobile robots," Mechatronics, vol. 19, no. 2, pp. 156-166, 2009.

[7] E. Kayacan, H. Ramon, and W. Saeys, "Adaptive neuro-fuzzy control of a spherical rolling robot using sliding-mode-controltheory-based online learning algorithm," IEEE Transactions on Systems, Man, and Cybernetics B: Cybernetics, vol. 43, 170, no. 1, p. 179, 2013.

[8] T. S. Li and Y. C. Huang, "MIMO adaptive fuzzy terminal sliding-mode controller for robotic manipulators," Information Sciences, vol. 180, no. 23, pp. 4641-4660, 2010.

[9] N. Yagiz and Y. Hacioglu, "Robust control of a spatial robot using fuzzy sliding modes," Mathematical and Computer Modelling, vol. 49, no. 1-2, pp. 114-127, 2009.

[10] Q. Zheng, Z. Chen, and Z. Gao, "A practical approach to disturbance decoupling control," Control Engineering Practice, vol. 17, no. 9, pp. 1016-1025, 2009.

[11] J. Han, "Auto-disturbance rejection control and its applications," Control and Decision, vol. 13, no. 1, pp. 19-23, 1998 (Chinese).

[12] J. Han, "A class of extended state observers for uncertain systems," Control and Decision, vol. 10, no. 1, pp. 85-88, 1995 (Chinese).

[13] J. Han, "From PID to active disturbance rejection control," IEEE Transactions on Industrial Electronics, vol. 56, no. 3, pp. 900906, 2009.

[14] Z. Gao, "Scaling and parameterization based controller tuning," in Proceedings of the American Control Conference, vol. 6, pp. 4989-4996, 2003.
[15] G. Tian and Z. Gao, "Frequency response analysis of active disturbance rejection based control system," in Proceedings of the 16th IEEE International Conference on Control Applications (CCA '07), pp. 1595-1599, October 2007.

[16] Y. Xia, Z. Zhu, M. Fu, and S. Wang, "Attitude tracking of rigid spacecraft with bounded disturbances," IEEE Transactions on Industrial Electronics, vol. 58, no. 2, pp. 647-659, 2011.

[17] Z. Zhu, Y. Xia, M. Fu, and S. Wang, "An observer-based missile guidance law," in Proceedings of the Chinese Control and Decision Conference (CCDC '11), pp. 1282-1287, Mianyang, China, May 2011.

[18] G. K. I. Mann, B. Hu, and R. G. Gosine, "Analysis of direct action fuzzy PID controller structures," IEEE Transactions on Systems, Man, and Cybernetics B: Cybernetics, vol. 29, no. 3, pp. 371-388, 1999.

[19] J. Carvajal, G. Chen, and H. Ogmen, "Fuzzy PID controller: design, performance evaluation, and stability analysis," Information Sciences, vol. 123, no. 3-4, pp. 249-270, 2000.

[20] H. B. Kazemian, "Comparative study of a learning fuzzy PID controller and a self-tuning controller," ISA Transactions, vol. 40, no. 3, pp. 245-253, 2001.

[21] M. Zhihong and X. Yu, "Adaptive terminal sliding mode tracking control for rigid robotic manipulators with uncertain dynamics," JSME International Journal C: Mechanical Systems, Machine Elements and Manufacturing, vol. 40, no. 3, pp. 493502, 1997. 

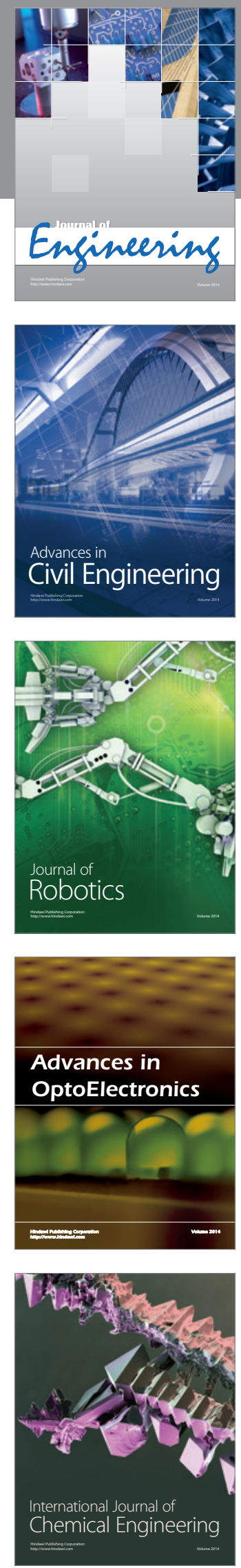

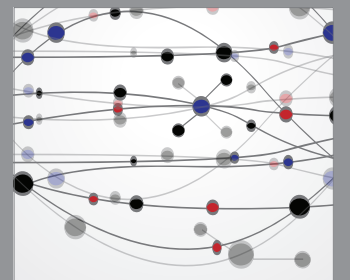

The Scientific World Journal
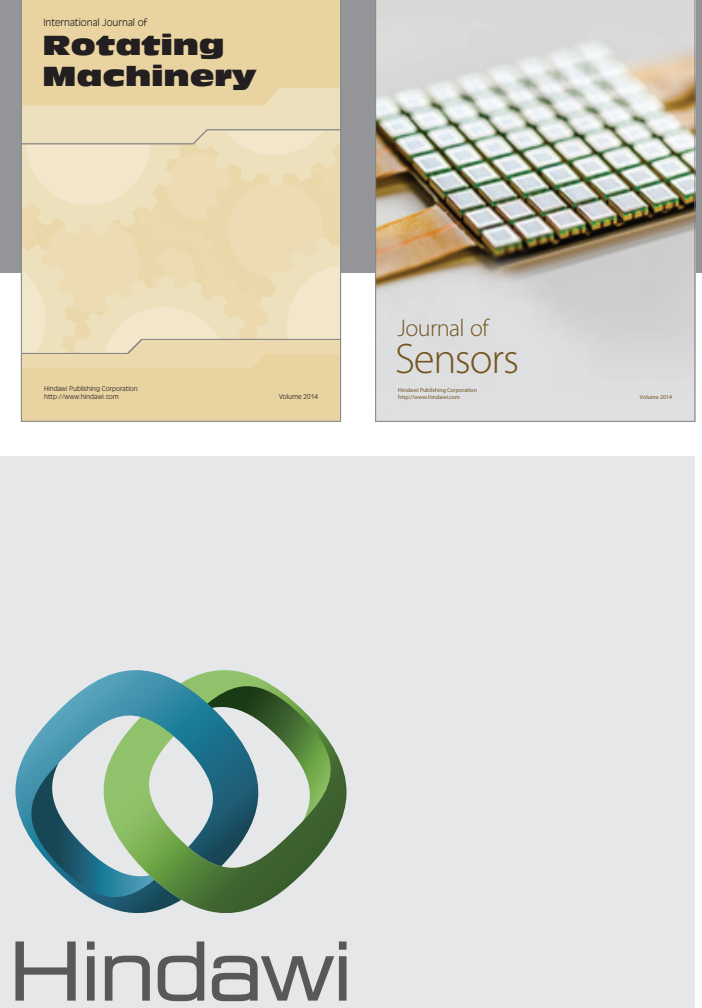

Submit your manuscripts at http://www.hindawi.com
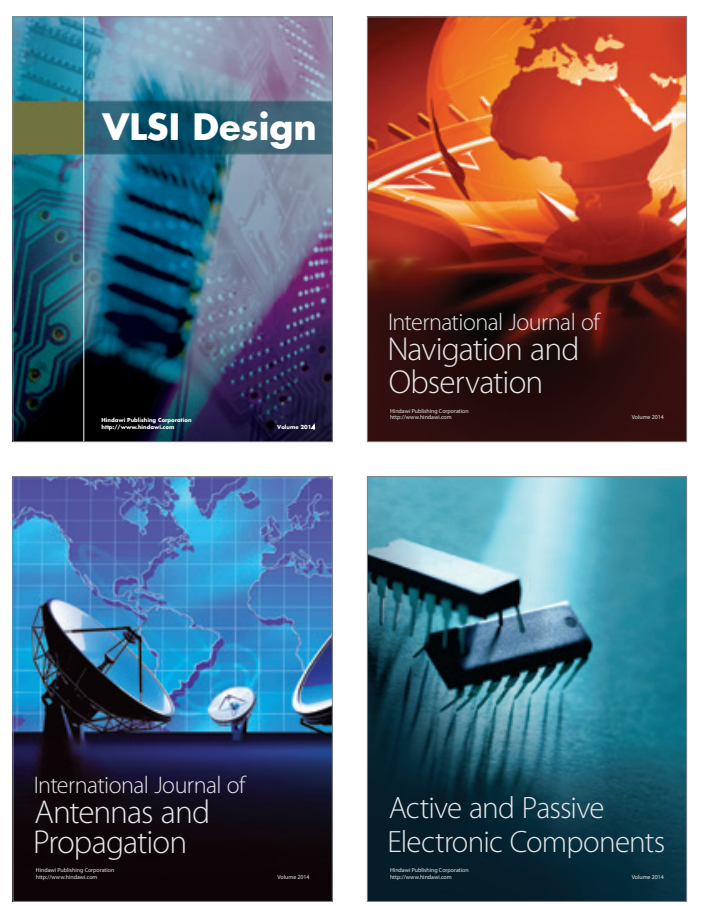
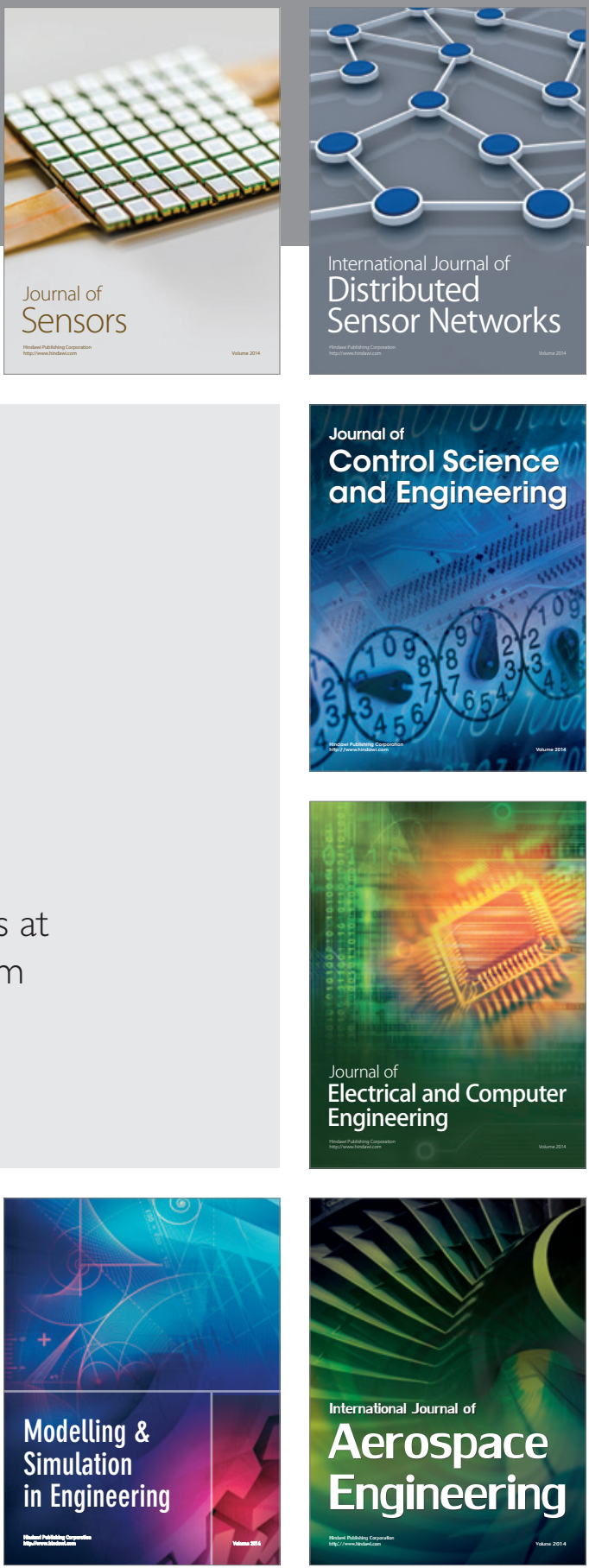

Journal of

Control Science

and Engineering
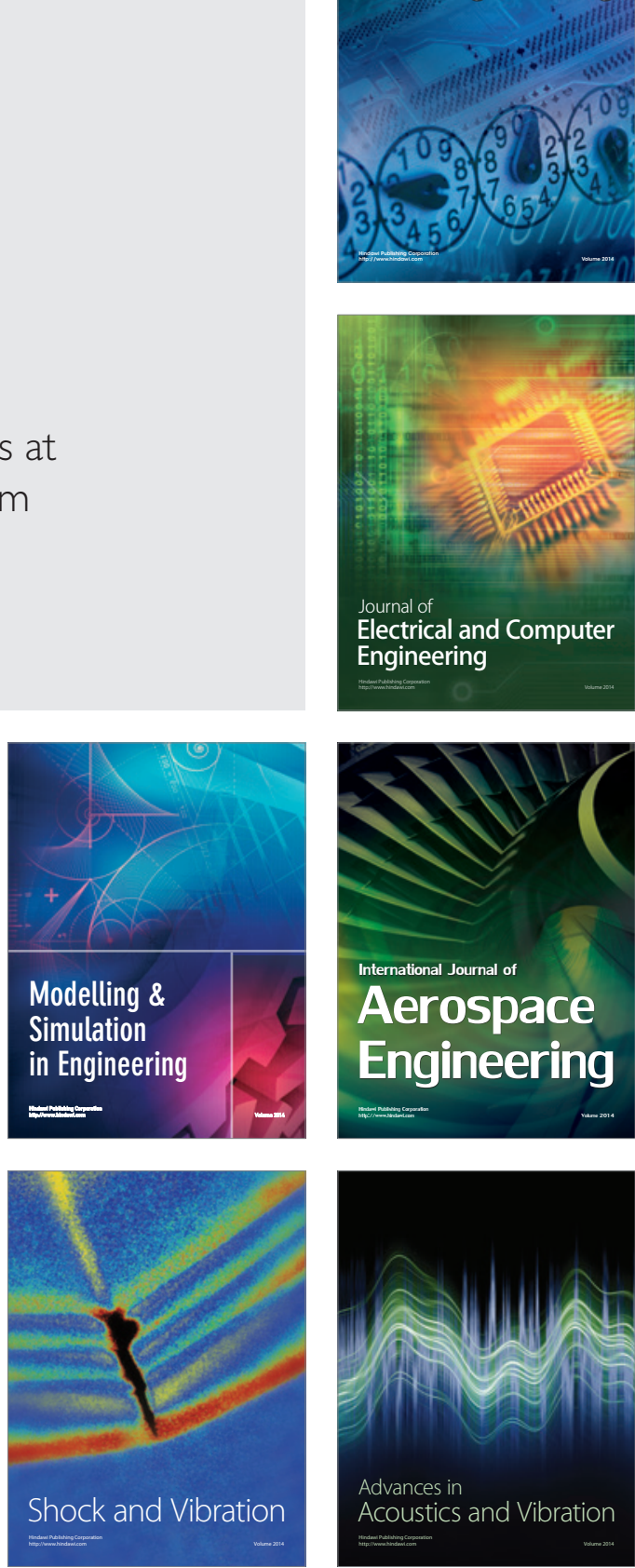